OPEN ACCESS

Edited by:

Alessandro Russo,

University of Pisa, Italy

Reviewed by:

Filippo Del Puente,

Ente Ospedaliero Ospedall

Galliera, Italy

Roger M. Echols,

Infectious Disease Drug Development

Consulting, LLC, United States

*Correspondence:

Tommaso Lupia

tommaso.lupia89@gmail.com

tThese authors have contributed equally to this work

Specialty section

This article was submitted to Infectious Diseases - Surveillance,

Prevention and Treatment,

a section of the journal

Frontiers in Medicine

Received: 14 October 2020 Accepted: 31 March 2021

Published: 07 May 2021

Citation:

Corcione S, Lupia T and De Rosa FG (2021) Novel Cephalosporins in Septic

Subjects and Severe Infections:

Present Findings and Future

Perspective. Front. Med. 8:617378.

doi: 10.3389/fmed.2021.617378

\section{Novel Cephalosporins in Septic Subjects and Severe Infections: Present Findings and Future Perspective}

\author{
Silvia Corcione ${ }^{1,2 t}$, Tommaso Lupia ${ }^{1 * t}$ and Francesco Giuseppe De Rosa ${ }^{1}$ \\ ${ }^{1}$ Department of Medical Sciences, Infectious Diseases, University of Turin, Turin, Italy, ${ }^{2}$ Tufts University School of Medicine, \\ Boston, MA, United States
}

In past decade, cephalosporins have developed significantly, and data regarding novel cephalosporins (i.e., ceftobiprole, ceftaroline, ceftolozane/tazobactam, ceftazidime/avibactam, and cefiderocol) within septic and bacteremic subjects are rising. These compounds generally offer very promising in vitro microbiological susceptibility, although the variability among gram-negative and -positive strains of different cohorts is noticed in the literature. We require further pharmacological data to measure the best dose in order to prevent sub-therapeutic drug levels in critically ill patients. These new compounds in theory are the sparing solution in the Enterobacteriales infection group for different antimicrobial classes such as aminoglycosides notably within endovascular and GNB-bacteremias, as well as colistin and carbapenem-sparing strategies, favoring good safety profile molecules. Moreover, new cephalosporins are the basis for the actual indications to open up new and exciting prospects for serious infections in the future. In future, patients will be addressed with the desirable approach to sepsis and serious infections in terms of their clinical situation, inherent features of the host, the sensitivity profile, and local epidemiology, for which evidence of the use of new cephalosporin in the treatment of severe infections will fill the remaining gaps.

Keywords: cephalosporin, sepsis, severe infections, blood-stream infections, multi-drug resistant bacteria

\section{INTRODUCTION}

Sepsis has been defined as "one of the oldest and most elusive syndromes in medicine" (1). In 2017 Rudd et al. estimated, worldwide, 48.9 million cases of sepsis and 11.0 million deaths due to sepsis (2). Sepsis complexity resides in a dysregulated host response to an infection and in jeopardy to develop acute organ dysfunction with a high risk of in-hospital mortality rates estimated between 25 and 30\% (3). This syndrome requires urgent treatment; thus, an awareness of the presenting characteristics of sepsis is highly important (4). In medicine, we must always look to the past with an eye to the future. From this perspective, this paper evaluates and discusses the available data on novel cephalosporins (i.e., ceftobiprole, ceftaroline, ceftolozane/tazobactam [C/T], ceftazidime/avibactam [C/A], and cefiderocol) (5) in the antimicrobial management of sepsis and severe infections to assess whether these new molecules can provide innovative answers to ancient questions (6). 


\section{CEPHALOSPORINS WITH A MAIN ANTI-MRSA ACTIVITY}

\section{Ceftobiprole}

Ceftobiprole medocaril is currently approved in Europe as an extended-spectrum cephalosporin for adult community acquired and nosocomial, non-ventilator-associated pneumonia (i.e., CAP and HAP) $(7,8)$, and skin and soft tissue infection (SSTIs), including diabetic foot infections $(9,10)$.

Clinical application and actual experiences with ceftobiprole are limited but nevertheless promising for sepsis and bloodstream infections (BSIs) $(11,12)$. A discreet proportion of gram-positive sepsis was included in phase III trials for CAP, HAP and SSTIs (7-10). Rello et al. (13) developed two interesting grouped analyses-a test of cure (TOC) and mortality-for ceftobiprole, vs. comparators (e.g., vancomycin, linezolid, and ceftazidime) for Staphylococcus spp. BSIs through the extrapolation of the clinical data directly from phase III studies (7-10). In the TOC analysis, the clinical cure rate of the ceftobiprole group (48.9 percent, 22/45 patients) was similar to that of the comparators (44.0 percent, 22/50), specifically the subgroups of coagulase-negative staphylococci (CoNS; 45.5 vs. $45.5 \%$ ), methicillin-sensitive Staphylococcus aureus (MSSA; 44.4 vs. 46.7\%), and methicillin-resistant S. aureus (MRSA; 55.6 vs. 22.2\%) (12, 13). Furthermore, the 30-day all-cause mortality in the ceftobiprole group was $8.9 \%(4 / 45)$ vs. $16.0 \%(8 / 50)$ in the comparator group $(12,13)$. In the ceftobiprole group, death rates were zero for MRSA bacteraemic patients compared with 22.2 per cent in the comparator cohorts $(12,13)$. Despite the interesting results, the conclusions drawn by Rello et al. had been obtained from a sample of just 18 patients, without a complete analysis of all data in the entire subgroup (13).

Ceftobiprole (alone or in combination) could therefore play an important role in treating endovascular infections due to its high bactericidal activity, favorable resistance profile, and potential synergism with other antigram-positive molecules (11-13). In a rat model of endocarditis, a subtherapeutic dose of ceftobiprole plus vancomycin was as effective against MRSA and vancomycin intermediate-resistant $S$. aureus strains as ceftobiprole standard dose monotherapy $(14,15)$. Theoretically, ceftobiprole used to treat endocarditis could permit a nephrotoxic-sparing strategy, thus avoiding the aminoglycoside and glycopeptide side effects and the need for therapeutic drug monitoring. Ceftobiprole also plays a vital role in penicillin-allergic subjects with severe grampositive infections (11-13). A daptomycin-based scheme, complemented with an adjunct of ceftobiprole, also seems promising in clinical applications within endocarditis therapeutic management $(16,17)$. An ongoing non-inferiority trial (double-blinded and randomized, https://clinicaltrials. gov/ct2/show/NCT03138733) comparing standard dose ceftobiprole and daptomycin $(6 \mathrm{mg} / \mathrm{kg} / 24 \mathrm{~h})$ in adult patients with $S$. aureus bacteraemia (including right-sided infective endocarditis) will provide more information (18). One potential concern surrounding ceftobiprole is determining the adequate dosage for bacteraemia and endocarditis. The prospect of accomplishing this target for MRSA strains with the ongoing approved dose is $>90 \%$ with MIC of 4 $\mathrm{mg} / \mathrm{L}(19-21)$.

A higher exposure $(100 \% \mathrm{~T}>\mathrm{MIC})$ is however associated with strong bactericidal action and therefore is the preferred goal for severe and high inoculum infections (19-21). With the current dose the probability of receiving $100 \%>\mathrm{T}>$ MIC for MRSA strains will be lower, but this can be improved significantly through the prolonged infusion (over $4 \mathrm{~h}$ ) or ongoing infusion of patients at higher doses $(500 \mathrm{mg} / 6 \mathrm{~h}$ or $1 \mathrm{~g} / 8-12 \mathrm{~h})(19-21)$.

Little is known about CSF penetration for ceftobiprole, which may still be a valuable option in primary meningitis caused by Streptococcus pneumoniae (including penicillinand ceftriaxone-resistant strains) and secondary, post-surgical meningitis requiring both gram-positive and susceptible gramnegative coverage $(11-13,22)$. The antibacterial activity was comparable to cefepime with $\beta$-lactamase-negative strains of Escherichia coli, Klebsiella pneumoniae, and Haemofilus influenzae in the animal model proposed by Stucki et al. (22). Moreover, the same authors (22) discovered that ceftobiprole reached about $16 \%$ of serum levels through inflamed meninges compared to about $2 \%$ of serum levels through uninflamed meninges in a rabbit meningitis model, similarly to cefepime pharmacokinetic in CSF.

This fifth-generation cephalosporin has been approved for official indications of CAP and HAP adults in 12 European countries, Canada, and Switzerland (11). Moreover, ceftobiprole blends excellent spectrum with beta-lactam safety for low to moderate MDR HAP pathogenes in frail patients who could be at great risk from adverse effects caused by MRSA or anti-MRSA coverage, including oxazolidinones or glycopeptides $(6,11,12)$.

There are promising activities of this novel cephalosporins on MRSA isolates, including Panton-Valentine Leukocidine positive strains, whether or not it is a slight change depending on the type of SCCmec, as shown in recent findings concerning ceftobiprole isolates in Phase III SSTI and pneumonia studies $(23,24)$. In severe adult CAP, coinfection, or superinfection over viral pneumonia, ceftobiprole can be a suitable option when the risk for MRSA or susceptible P.aeruginosa coinfection is high $(6,11,12)$.

VAP still represents an area of uncertainty according to clinical trial results (8) due to the inadequate sample size and substantial baseline sample heterogeneous features (6). Ceftobiprole data for BSIs in real life are limited, but promising, despite the need for further pharmacokinetic data in special populations such as patients with critical illness or elevated creatinine clearance (25).

\section{Ceftaroline}

Ceftaroline is a fifth-generation cephalosporin with a peculiar affinity to the penicillin binding protein (PBPs) 2a, an MRSAspecific protein, with an excellent spectrum of activity on common bacterial causes of CAP $(26,27)$ and SSTIs (28). Ceftaroline has activity against a wide spectrum of grampositive bacteria including MSSA and MRSA, also including 
some resistant $S$. aureus strains (vancomycin intermediate, heterogeneous vancomycin intermediate, vancomycin-resistant, or daptomycin non-susceptible) and MDR S. pneumoniae (29-31). Moreover, ceftaroline also exhibits activity against a broad group of gram-negative, ESBL-negative, or AmpCproducing Enterobacteriaceae (29-31). The US Food and Drug Administration (FDA) approved a label expansion for the treatment of $S$. aureus bacteraemia associated with SSTIs in adults in 2015 and in the pediatric population in 2016 (32). The literature regarding the bacteraemic cohorts of patients treated with ceftaroline (alone or in combination) is growing and has been mostly comprised of subjects with a diagnosis of either persistent bacteraemia or MRSA bacteraemia that is not susceptible to vancomycin or daptomycin (32-47). Persistent bacteraemia (with or without a known focus of infections) is not an uncommon finding, which may be associated with increased mortality and morbidity, and refers to blood cultures that were positive within the same infectious episode and on different days $(33,34)$. Interesting observations on time to eradication of MRSA in BSIs were extrapolated from a retrospective matched case-control study by Paladino et al. (33): the ceftaroline cohort reported a median time to eradication that was about half the median time of the control group treated with vancomycin (4 vs. 8 days; interquartile range [IQR]: $3.0-7.5$ days vs. $5.8-19.5$ days; $P=0.02)$. The time to eradication was further reduced to 2 days (IQR: 1-4 days) in Arshad et al.'s (34) multicenter observational study of 211 patients (33). Clinical success in the most representative studies has ranged from 60 to $88 \%$ (3439). Interestingly, Arshad et al. (34) reported a clinical cure rate of $69.7 \%$ when ceftaroline was used as a monotherapy and $64.9 \%$ when it was used in combination. Furthermore, even a microbiological cure reported good results, between 70 and $100 \%$, but these were variable depending on the time of consideration (34). Mootz et al.'s (45) retrospective comparative effectiveness study included adults hospitalized with sepsis who received ceftaroline or daptomycin within 14 days of hospital admission. Patients treated with ceftaroline were less likely to experience readmission at 30 days (25 vs. $37 \%, P=0.06$ ), 60 days (27 vs. $44 \%, P=0.008$ ) and 90 days (28 vs. $46 \%, P=$ 0.01 ) compared to those treated with daptomycin (45). Moreover, the ceftaroline group showed a lower in-hospital mortality rate (7 vs. $12 \%, P=0.4$ ) at 30 days ( 3 vs. $9 \%, P=0.1$ ), 60 days (6 vs. $12 \%, P=0.2$ ), and 90 days ( 7 vs. $15 \%, P=0.1$ ) vs. the comparator (45).

Ceftaroline, a rapid BSI clearance beta-lactam, also serves as an interesting solution to complicated endocarditis (46). This is illustrated by the retrospective CAPTURE analysis involving 55 patients receiving ceftaroline treatment with gram-positive endocarditis (47). The overall success rate in this study was $70.9 \%$ (47). In particular, ceftaroline therapies were extremely effective as a first-line therapy (75.0\%) as well as in patients suffering from right-sided endocarditis (80.8\%) and MRSA (77.3\%) (47).

Ceftaroline is currently used in the management of persistent gram-positive bacteraemia (36) and, also due to the wide spectrum of microbiological activity comprising gram-negative organisms, may be a feasible choice for catheter-related and -associated BSIs $(12,32)$. The bactericidal and timedependent activity of ceftaroline, as well as ceftobiprole, could potentially be implemented in therapeutic schemes for bacteraemia and endovascular infection to improve the safety of kidney function, limiting glycopeptides and the need for therapeutic monitoring, with consistent advantages in centers with scarce laboratory resources $(11,12,32,33)$. Ceftaroline could also be used in the treatment of severe infections, including both primary (e.g., post-traumatic) and secondary (e.g., post-surgical) bacterial meningitis, although few data are available for CSF drug concentrations $(48,49)$.

Stucki et al. (48) estimated that $15 \%$ of the serum level of CSF penetrated inflammatory meninges and about $3 \%$ of non-inflamed meninges. Mermer et al. (49) found that in an experimental meningitis model both ceftaroline and vancomycin have similar antibacterial efficacy in treating MRSA. Pani et al. (32) confirmed the use of ceftaroline as the fifth off-label indication for meningitis in their latest systematic research.

\section{CEPHALOSPORINS WITH MAIN ANTI-GRAM-NEGATIVE ACTIVITY \\ Ceftolozane/Tazobactam}

$\mathrm{C} / \mathrm{T}$ is a combination of the renowned $\beta$-lactamase inhibitor tazobactam and an innovative anti-pseudomonal cephalosporin $(5,50)$. The FDA and the EMA have approved $\mathrm{C} / \mathrm{T}$ for complicated urinary tract infection (cUTI) (51) and complicated intra-abdominal infection (cIAI) (52) at a dose of $1.5 \mathrm{~g}$ (ratio of 1.0 ceftolozane to 0.5 tazobactam) every $8 \mathrm{~h}$, with double dosage $(3 \mathrm{~g}$; ratio of 2.0 ceftolozane to 1.0 tazobactam) in the phase III study ASPECT-NP for the treatment of NP (53). C/T is active in several multidrug resistant (MDR) and extensively drug-resistant (XDR) Enterobacteriales strains, including ESBL-producting strains and P. aeruginosa. (54).

Regarding ESBL strains, $\mathrm{C} / \mathrm{T}$ has differences depending on the pathogen concerned. $\mathrm{C} / \mathrm{T}$, in a study published by Tato et al. (55), seems to have higher activity against ESBL-producing E. coli than Klebsiella spp. Moreover, among ESBL pathogens, Castanheira et al. (56) have found that $\mathrm{C} / \mathrm{T}$ retains a lower activity in bla $a_{\mathrm{SHV}}$ isolates $(61.1 \%)$ than bla $a_{\mathrm{CTX}-\mathrm{M}}$ strains $(91.2 \%)$. All together, these data show that $\mathrm{C} / \mathrm{T}$ is a major component of a carbapenem-sparing strategy, at least in the empiric setting, even if more evidence is needed to confirm the exact role as a targeted treatment of ESBL infections (57).

An increased risk of hospital mortality has been linked to inadequate initial antibiotic treatment for Pseudomonas aeruginosa BSI (58). From a microbiological perspective, 88 percent $(n=615)$ of isolates were susceptible to $\mathrm{C} / \mathrm{T}$ in a major multi-center study carried out between 2012 and 2015 for meropenem-non-susceptible $P$. aeruginosa isolates in 32 US medically-based centers (59).

For contrast, additional single-center surveillance studies have shown a 60-94 percent $\mathrm{C} / \mathrm{T}$ susceptibility of different $P$. aeruginosa isolate populations (60-62). 
From a clinical point of view, Bassetti et al. (63) described one of the largest clinical trials using $\mathrm{C} / \mathrm{T}$ in a multicenter cohort of 101 patients with documented $P$. aeruginosa infection. Sepsis and septic shock were present at diagnosis in 26.7 and $11.9 \%$ of patients, respectively, and concomitant $P$. aeruginosa bacteraemia was ruled out in only $15.8 \%$ of subjects (63).

The only independent predictor of clinical failure was sepsis (odds ratio $[\mathrm{OR}]=3.02,95 \%$ confidence interval $[\mathrm{CI}]$ : 1.01-9.2; $P=0.05)$ for patients with clinical success in comparisons to those suffering a clinical failure with multivariate analyses (odds ratio $[\mathrm{OR}]=3.02,95$ per cent $\mathrm{CI}$ : $[\mathrm{CI}]: 1.01-9.2 ; P=0.05)(63)$.

All together, these data show that $\mathrm{C} / \mathrm{T}$ may be a valuable option to prevent nephrotoxicity with colistinor aminoglycoside-sparing regimens including the risk of subtherapeutic dosages associated with reduced renal clearances $(64,65)$.

CEFTABUSE register (65) results showed a non-significant trend toward more favorable 14-day clinical cure rates in $\mathrm{C} / \mathrm{T}$ patients than aminoglycoside or colistin (81.3 vs. $56.3 \%$; $P=0.11 \%)$. A similar pattern was found for crude deaths of 30 days ( 18.8 vs. $28.1 \% ; P=0.73$ ) and acute kidney injury prevalence ( 0.0 vs. $25.0 \%$; and $P=0.04$ ), favors $\mathrm{C} / \mathrm{T}$ vs. colistin or aminoglycoside.

Similarly, a retrospective multicenter observational cohort study [644] also found that $\mathrm{C} / \mathrm{T}$ administration was independently associated with clinical cure (adjusted OR: 2.63; 95\% CI: 1.31-5.30) and protected against AKI (adjusted OR: 0.08 ; 95\% CI: $0.03-0.22$ ) without any difference in in-hospital mortality. In addition, a systematic Maraolo et al. (62) study concluded that the therapy $\mathrm{C} / \mathrm{T}$ could be useful even outside of an accepted setting of indication for difficult-to-treat $P$. aeruginosa infections: BSI is the third commonly indicated off-label (23/130; 17.7\%).

\section{Ceftazidime/Avibactam}

$\mathrm{C} / \mathrm{A}$ is an intravenous combination of a third-generation cephalosporin with the non- $\beta$-lactam $/ \beta$-lactamase inhibitor avibactam (66), with activity against ESBL-producing bacteria, $P$. aeruginosa and KPC or OXA-48 carbapenemase producing bacteria. C/A has been approved in cIAI (67), cUTI (68), and NP (69) therapy as well as infection by microorganisms resistant to ceftazidime, according to a specific trial (RECLAIM, RECAPTURE, REPROVE, and REPRISE study, respectively) and, given the comparator mainly based on carbapenem drug administration, it is also a major component of a carbapenemsparing strategy. Moreover, real-life data did show clinical efficacy in patients with KPC and OXA-48 carbapenemases. In a prospective study, Sousa et al. (70) described a cohort of 55 patients, $54 \%$ of whom presented with severe septic shock or sepsis; moreover, 54 of the 57 isolates were OXA-48producing K. pneumoniae, $46 \%$ (26/55) of patients had confirmed bacteraemia, and C/A was mainly given as monotherapy (81\%) with a mean duration of 13 days; The 14-day mortality rate was $14 \%$ (70). In systematic reviews and meta-analysis with infections by carbapenem-resistant bacteria, Fiore et al. (71) did not observe any difference in the mortality rates between $\mathrm{C} / \mathrm{A}$ monotherapy or combination therapy $(N=503$ patients; direct evidence OR: 0.96; 95\% CI: 0.65-1.41), with similar findings in the unregistered systematic review by Onorato et al. (72).

A retrospective longitudinal study of 138 patients with KPC$\mathrm{Kp}$ bacteraemia, in which a significantly lower mortality was observed in any patient with C/A than other drugs (36.5 vs. $55.8 \%, P=0.05)$, and in which this was the only factor that was substantially correlated with survival, was addressed by Tumbarello et al. (73). In this complex analysis, the authors identified septic shock, neutropenia, Charlson Comorbidity Index $\geq 3$, and recent mechanical ventilation as independent predictors of mortality, whereas C/A was the sole independent predictor of survival (73). In addition, Shields confirmed higher rates of clinical success $(P=0.006)$ and survival $(P=0.01)$ for $\mathrm{C} / \mathrm{A}$ compared to other regimens and observed higher renal safety compared to aminoglycoside and colistin-containing regimens $(P=0.002)(74)$.

All together, data from official trials and real-life experiences showed that $\mathrm{C} / \mathrm{A}$ is representing a major component in carbapenem-sparing strategies, including patients infected by KPC or OXA-48 carbapenemases producing bacteria in addition to $P$. aeruginosa and ESBL-producing bacteria (75).

\section{Cefiderocol}

Cefiderocol is an innovative siderophore cephalosporin that was produced to target carbapenem-resistant pathogens, including fermenting and non-fermenting (i.e., P. aeruginosa and Acinetobacter baumannii) GNB $(76,77)$. Among such pathogens is also included Stenotrophomonas maltophilia, which is intrinsically resistant to carbapenems $(76,78)$. In the CREDIBLE CR, cefiderocol was studied in a randomized, open-label, prospective, phase III clinical study in individuals with carbapenem-resistant gram-negative bacteria infections, regardless of species or infection site source and including sepsis and BSIs (ClinicalTrials.gov registration: NCT02714595) $(79,80)$. The clinical cure between cefiderocol and the comparator, defined as best available therapy (BAT), was similar for NP (50 vs. 53\%) and BSI (43 vs. 43\%). Besides, in complicated UTIs, cefiderocol was not inferior to the BAT group in microbiological eradication (53 vs. 20\%) (80). In the cefiderocol group, more numerical deaths occurred, especially in the Acinetobacter spp subset, a finding which was not unequivocally explained. These results endorse cefiderocol as an alternative for treating patients with limited treatment options of carbapenem-resistant infections (80).

Wunderink et al. (81) recently published an APEKS-NP randomized double-blind, phase III, non-inferiority analysis in which 148 subjects and 152 subjects were allocated, respectively, for cefiderocol and meropenem. The authors suggested that cefiderocol was non-inferior in patients with Gram-negative NP equal to high dose extended-infusion meropenem and similar for all cases-mortality on Day 14 (12.4 vs. 11.6\%) (81). The findings indicate that cefiderocol is a promising solution for treating NP, including those caused by MDR gram-negative organisms (81). 

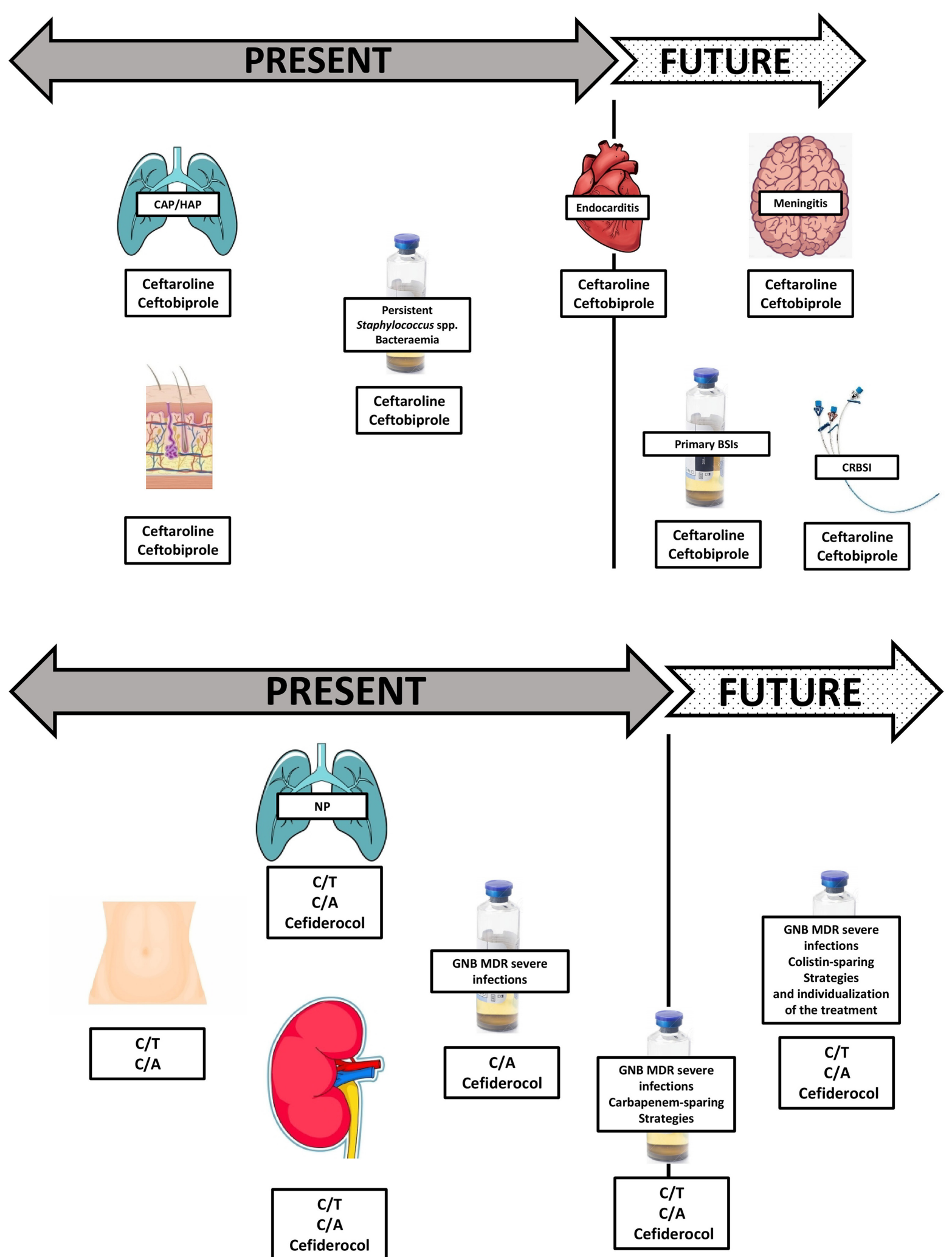

FIGURE 1 | Present and future perspectives within novel cephalosporins compounds. 
TABLE 1 | Advantages of monotherapy and combination antimicrobial therapy.

\begin{tabular}{ll}
\hline PROs of Monotherapy & PROs of Combination Therapy \\
\hline Low antibiotic pressure & $\begin{array}{l}\text { Avoid resistance development in } \\
\text { difficult-to-treat infections } \\
\text { Active on different mechanism }\end{array}$ \\
Improve de-escalation approach & $\begin{array}{l}\text { In MDR infections to ensure sensitivity } \\
\text { Improve antibiotic stewardship bundle }\end{array}$ \\
$\begin{array}{l}\text { Accelerate pathogen clearance high } \\
\text { Reduce the risk of antibiotic }\end{array}$ & $\begin{array}{l}\text { Improve synergy between molecules } \\
\text { antagonism }\end{array}$ \\
Improve diagnosis & $\begin{array}{l}\text { Decrease the risk of inappropriate } \\
\text { empiric antibiotic therapy }\end{array}$
\end{tabular}

Hsueh et al. (82) measured in vitro cefiderocol, C/T, and C/A microbiological profile for $P$. aeruginosa and S. maltophilia and A.baumannii isolates in the bloodstream. In comparison with the $\mathrm{C} / \mathrm{T}$ and $\mathrm{C} / \mathrm{A}$, Cefiderocol demonstrated much greater in vitro activity with MICs $\leq 4 \mathrm{mg} / \mathrm{L}$ for $P$. aeruginosa isolates resistant to colistin or imipenem (82).

Presently, in order to improve therapeutic effectiveness in serious CRE infections, high levels and combining methods which may have a new inhibitor $\beta$-lactam/ $\beta$-lactamase are likely to be considered $(77,78,83)$. Cefiderocol has been added to these therapeutic options, increasing the antimicrobial spectrum to A. baumannii and S.maltophilia, which in the last decade have been frequently omitted from the new molecules, theoretically allowing for enhanced individualization based on molecular resistance phenotypes, disease severity, susceptibility profiles, and patient characteristics in antimicrobial strategies $(77,78,83,84)$.

Besides, cefiderocol, from a pharmacokinetic point of view, is unique for its dosing regimen to include patients with an augmented renal clearance which is the primary cause for underdosing in very sick patients for beta-lactams.

\section{DISCUSSION}

The creation of three fifth-generation cephalosporins has made a major evolution in the last decade (i.e., ceftobiprole, ceftaroline, and ceftolozane), along with the development of cefiderocol, with its new "trojan horse" active transport mechanism for entering multi-drug resistant bacteria, as well as through novel therapeutic binomials (C/T and C/A) $(5,11,44,79)$. Data on novel cephalosporins may also be available, in addition to the official registration trials, in patients with bloodstream infections and severe infections as well as in infections by MDR bacteria including carbapenemases-producing bacteria from real-life settings. In addition, the future of antimicrobial stewardship in septic and bacteremic patients can be assessed through a multilevel evaluation from the microbiological, pharmacological, clinical, and financial perspectives $(5,12,77,78)$. Although the literature is clear on specific variability in gram-negative and gram-positive strains from various countries and cohorts, as well as different rates of carbapenem resistance, the in vitro susceptibilities of the novel cephalosporins are generally very promising. $(5,12,77,78)$. This variability makes it crucial to know their global and local epidemiologies, particularly in MDR gram-negatives with limited treatment options. Furthermore, there is a critical need for more pharmacological data to assess the best dosage and administration modalities in critically ill patients in order to avoid subtherapeutic levels of the $\operatorname{drug}(5,12,77,78)$.

Theoretically, such new compounds allow a sparing approach in different antimicrobial classes, such as carbapenems, aminoglycosides, colistin, and also vancomycin for ceftaroline and ceftobiprole $(12,65,77,78)$. We are presented with molecules with an increasingly strong pathogen-specific identity, such as C/T for MDR/XDR P. aeruginosa and cefiderocol for difficult-totreat CR strains, and we face a great change in the management of MRSA and endovascular bacteremia due to the evolving clinical data on ceftaroline and ceftobiprole $(5,11,44,79)$. Time seems to be on the side of these novel cephalosporins, making them useful in several severe infections (Figure 1). These molecules can also be used in therapeutic combinations, under particular circumstances. The primary rationale for using two distinct classes of antibiotics with activity against a single pathogen is, on the one hand, to potentiate pathogen clearance and, on the other, to assure the pathogen's susceptibility to the empiric therapy $(85,86)$. Conversely, monotherapy reduces the risk of antibiotic pressure, the rate of new infections, antibiotic antagonism, toxicity, and costs, though it may not cover MDR pathogens (Table 1) $(85,86)$. Combination antibiotic treatment was recommended in international guidelines for primary management of septic shock to provide appropriate empirical antibiotic coverage in a scenario with high risk of MDR pathogens (87). However, several other studies found no superiority of combination treatment, and some analyses showed an increased rate of side effects in patients receiving combination therapy (88-90). The desired approach to sepsis and serious infections would be presented to patients in the future based on their clinical condition, host characteristics, susceptibility profiles, and local epidemiology, which would fill the gaps in the use of new cephalosporins that currently exist. Finally, literature data emphasize the small spread of every study and also stress the importance of local monitoring. When determining early use of these agents in severely ill patients, careful consideration should be given to local susceptibility patterns.

\section{DATA AVAILABILITY STATEMENT}

The original contributions presented in the study are included in the article/supplementary material, further inquiries can be directed to the corresponding author/s.

\section{AUTHOR CONTRIBUTIONS}

All authors listed have made a substantial, direct and intellectual contribution to the work, and approved it for publication. 


\section{REFERENCES}

1. Angus DC, van der Poll T. Severe sepsis and septic shock. N Engl J Med. (2013) 369:2063. doi: 10.1056/NEJMc1312359

2. Rudd KE, Johnson SC, Agesa KM, Shackelford KA, Tsoi D, Kievlan DR, et al. Global, regional, and national sepsis incidence and mortality, 1990-2017: analysis for the Global Burden of Disease Study. Lancet. (2020) 395:20011. doi: 10.1016/S0140-6736(19)32989-7

3. Cecconi M, Evans L, Levy M, Rhodes A. Sepsis and septic shock. Lancet. (2018) 392:75-87. doi: 10.1016/S0140-6736(18)30696-2

4. Cohen J, Vincent JL, Adhikari NK, Machado FR, Angus DC, Calandra T, et al. Sepsis: a roadmap for future research. Lancet Infect Dis. (2015) 15:581614. doi: 10.1016/S1473-3099(15)70112-X

5. Lupia T, Corcione S, Mornese Pinna S, De Rosa FG. New cephalosporins for the treatment of pneumonia in internal medicine wards. J Thorac Dis. (2020) 12:3747-63. doi: 10.21037/jtd-20-417

6. Fleischmann C, Scherag A, Adhikari NK, Hartog CS, Tsaganos T, Schlattmann $\mathrm{P}$, et al. International forum of acute care trialists. assessment of global incidence and mortality of hospital-treated sepsis. current estimates and limitations. Am J Respir Crit Care Med. (2016) 193:25972. doi: 10.1164/rccm.201504-0781OC

7. Nicholson SC, Welte T, File TM Jr, Strauss RS, Michiels B, Kaul P, et al. A randomised, double-blind trial comparing ceftobiprole medocaril with ceftriaxone with or without linezolid for the treatment of patients with community-acquired pneumonia requiring hospitalisation. Int J Antimicrob Agents. (2012) 39:240-6. doi: 10.1016/j.ijantimicag.2011.11.005

8. Awad SS, Rodriguez AH, Chuang YC, Marjanek Z, Pareigis AJ, Reis G, et al. A phase III randomized double-blind comparison of ceftobiprole medocaril versus ceftazidime plus linezolid for the treatment of hospital-acquired pneumonia. Clin Infect Dis. (2014) 59:51-61. doi: 10.1093/cid/ciu219

9. Noel GJ, Bush K, Bagchi P, Ianus J, Strauss RS. A randomized, double-blind trial comparing ceftobiprole medocaril with vancomycin plus ceftazidime for the treatment of patients with complicated skin and skin-structure infections. Clin Infect Dis. (2008) 46:647-55. doi: 10.1086/526527

10. Noel GJ, Strauss RS, Amsler K, Heep M, Pypstra R, Solomkin JS. Results of a double-blind, randomized trial of ceftobiprole treatment of complicated skin and skin structure infections caused by gram-positive bacteria. Antimicrob Agents Chemother. (2008) 52:37-44. doi: 10.1128/AAC.00551-07

11. Giacobbe DR, De Rosa FG, Del Bono V, Grossi PA, Pea F, Petrosillo N, et al. Ceftobiprole: drug evaluation and place in therapy. Expert Rev Anti Infect Ther. (2019) 17:689-98. doi: 10.1080/14787210.2019.1667229

12. Soriano A, Morata L. Ceftobripole: experience in staphylococcal bacteremia. Revista Espanola de Quimioterapia. (2019) 32(Suppl. 3):24-8.

13. Rello J, Rahav G, Scheeren T, Saulay M, Engelhardt M, Welte T. A pooled analysis of clinical cure and mortality with ceftobiprole medocaril versus comparators in staphylococcal bacteraemia in complicated skin infections, and community- and hospital-acquired pneumonia. ECCMID. (2016). O-318.

14. Fernandez J, Abbanat D, Shang W, He W, Amsler K, Hastings J, et al. Synergistic activity of ceftobiprole and vancomycin in a rat model of infective endocarditis caused by methicillin-resistant and glycopeptideintermediate Staphylococcus aureus. Antimicrob Agents Chemother. (2012) 56:1476-84. doi: 10.1128/AAC.06057-11

15. Entenza JM, Veloso TR, Vouillamoz J, Giddey M, Majcherczyk P, Moreillon P. In vivo synergism of ceftobiprole and vancomycin against experimental endocarditis due to vancomycin-intermediate Staphylococcus aureus. Antimicrob Agents Chemother. (2011) 55:3977-84. doi: 10.1128/AAC.00402-11

16. Dhand A, Bayer AS, Pogliano J, Yang SJ, Bolaris M, Nizet V, et al. Use of antistaphylococcal beta-lactams to increase daptomycin activity in eradicating persistent bacteremia due to methicillin-resistant Staphylococcus aureus: role of enhanced daptomycin binding. Clin Infect Dis. (2011) 53:15863. doi: 10.1093/cid/cir340

17. Moise PA, Amodio-Groton M, Rashid M, Lamp KC, HoffmanRoberts HL, Sakoulas G, et al. Multicenter evaluation of the clinical outcomes of daptomycin with and without concomitant $\beta$-lactams in patients with Staphylococcus aureus bacteremia and mild to moderate renal impairment. Antimicrob Agents Chemother. (2013) 57:1192-200. doi: 10.1128/AAC.02192-12
18. https://clinicaltrials.gov/ct2/show/NCT03138733

19. Zhanel GG, Voth D, Nichol K, Karlowsky JA, Noreddin AM, Hoban DJ. Pharmacodynamic activity of ceftobiprole compared with vancomycin versus methicillin-resistant Staphylococcus aureus (MRSA), vancomycinintermediate Staphylococcus aureus (VISA) and vancomycin-resistant Staphylococcus aureus (VRSA) using an in vitro model. J Antimicrob Chemother. (2009) 64:364-9. doi: 10.1093/jac/dkp176

20. Kimko H, Xu X, Nandy P, Samtani MN, Strauss RS, Bagchi P, et al. Pharmacodynamic profiling of ceftobiprole for treatment of complicated skin and skin structure infections. Antimicrob Agents Chemother. (2009) 53:33714. doi: 10.1128/AAC.01653-08

21. Torres A, Mouton JW, Pea F. Pharmacokinetics and dosing of ceftobiprole medocaril for the treatment of hospital- and community-acquired pneumonia in different patient populations. Clin Pharmacokinet. (2016) 55:150720. doi: 10.1007/s40262-016-0418-Z

22. Stucki A, Cottagnoud M, Acosta F, Egerman U, Läuffer J, Cottagnoud P. Evaluation of ceftobiprole activity against a variety of gram-negative pathogens, including Escherichia coli, Haemophilus influenzae ( $\beta$-lactamase positive and $\beta$-lactamase negative), and Klebsiella pneumoniae, in a rabbit meningitis model. Antimicrob Agents Chemother. (2012) 56:9215. doi: 10.1128/AAC.01537-10

23. Davies TA, Shang W, Amsler KM, Bajaksouzian S, Jacobs MR, Bush K. Molecular characterisation of meticillin-resistant Staphylococcus aureus isolates from two ceftobiprole Phase III complicated skin and skinstructure infection clinical trials. Int J Antimicrob Agents. (2009) 34:1668. doi: 10.1016/j.ijantimicag.2009.02.013

24. Mendes RE, Deshpande LM, Costello AJ, Farrell DJ, Jones RN, Flamm RK. Genotypic characterization of methicillin-resistant Staphylococcus aureus recovered at baseline from Phase III pneumonia clinical trials for ceftobiprole. Microb Drug Resist. (2016) 22:53-8. doi: 10.1089/mdr.2014.0307

25. Azanza Perea JR, Sádaba Díaz de Rada B. Ceftobiprole: pharmacokinetics and PK/PD profile. Rev Esp Quimioter. (2019) 32 (Suppl 3):11-6.

26. File TM Jr, Low DE, Eckburg PB, Talbot GH, Friedland HD, Lee J, et al. FOCUS 1 investigators. FOCUS 1: a randomized, double-blinded, multicentre, Phase III trial of the efficacy and safety of ceftaroline fosamil versus ceftriaxone in community-acquired pneumonia. J Antimicrob Chemother. (2011) 66(Suppl. 3):iii19-32. doi: 10.1093/jac/dkr096

27. Low DE, File TM Jr, Eckburg PB, Talbot GH, David Friedland $H$, Lee J, et al. FOCUS 2 investigators. FOCUS 2: a randomized, doubleblinded, multicentre, Phase III trial of the efficacy and safety of ceftaroline fosamil versus ceftriaxone in community-acquired pneumonia. J Antimicrob Chemother. (2011) 66(Suppl. 3):iii33-44. doi: 10.1093/jac/d $\mathrm{kr} 097$

28. Scott LJ. Ceftaroline fosamil: a review in complicated skin and soft tissue infections and community-acquired pneumonia. Drugs. (2016) 76:165974 doi: 10.1007/s40265-016-0654-4

29. Zhanel GG, Sniezek G, Schweizer F, Zelenitsky S, Lagacé-Wiens PR, Rubinstein E, et al. Ceftaroline: a novel broad-spectrum cephalosporin with activity against meticillin-resistant Staphylococcus aureus. Drugs. (2009) 69:809-31. doi: 10.2165/00003495-200969070-00003

30. Jorgenson MR, DePestel DD, Carver PL. Ceftaroline fosamil: a novel broad-spectrum cephalosporin with activity against methicillin-resistant Staphylococcus aureus. Ann Pharmacother. (2011) 45:1384-98. doi: 10.1345/aph.1Q225

31. Lodise TP, Low DE. Ceftaroline fosamil in the treatment of community-acquired bacterial pneumonia and acute bacterial skin and skin structure infections. Drugs. (2012) 72:1473-93. doi: 10.2165/11635660-000000000-00000

32. Pani A, Colombo F, Agnelli F, Frantellizzi V, Baratta F, Pastori D, et al. Off-label use of ceftaroline fosamil: a systematic review. Int J Antimicrob Agents. (2019) 54:562-71. doi: 10.1016/j.ijantimicag.2019.06.025

33. Paladino JA, Jacobs DM, Shields RK, Taylor J, Bader J, Adelman MH, et al. Use of ceftaroline after glycopeptide failure to eradicate meticillinresistant Staphylococcus aureus bacteraemia with elevated vancomycin minimum inhibitory concentrations. Int J Antimicrob Agents. (2014) 44:55763. doi: 10.1016/j.ijantimicag.2014.07.024

34. Arshad S, Huang V, Hartman P, Perri MB, Moreno D, Zervos MJ. Ceftaroline fosamil monotherapy for methicillin-resistant Staphylococcus 
aureus bacteremia: a comparative clinical outcomes study. Int J Infect Dis. (2017) 57:27-31. doi: 10.1016/j.ijid.2017.01.019

35. Ho TT, Cadena J, Childs LM, Gonzalez-Velez M, Lewis JS. Methicillinresistant Staphylococcus aureus bacteraemia and endocarditis treated with ceftaroline salvage therapy. J Antimicrob Chemother. (2012) 67:126770. doi: $10.1093 / \mathrm{jac} / \mathrm{dks} 006$

36. Gritsenko D, Fedorenko M, Ruhe JJ, Altshuler J. Combination therapy with vancomycin and ceftaroline for refractory methicillin-resistant Staphylococcus aureus bacteremia: a case series. Clin Ther. (2017) 39:212-8. doi: 10.1016/j.clinthera.2016.12.005

37. Karki A, Thurm C, Cervellione K. Experience with ceftaroline for treatment of methicillin-resistant Staphylococcus aureus pneumonia in a community hospital. J Community Hosp Intern Med Perspect. (2017) 7:3002. doi: $10.1080 / 20009666.2017 .1374107$

38. Zasowski EJ, Trinh TD, Claeys KC, Casapao AM, Sabagha N, Lagnf AM, et al. Multicenter observational study of ceftaroline fosamil for methicillin-resistant Staphylococcus aureus bloodstream infections. Antimicrob. Agents Chemother. (2017) 61:e02015-16. doi: 10.1128/AAC.02015-16

39. Polenakovik HM, Pleiman CM. Ceftaroline for methicillin-resistant Staphylococcus aureus bacteraemia: case series and review of the literature. Int J Antimicrob Agents. (2013) 42:450-5 doi: 10.1016/j.ijantimicag.2013.07.005

40. Casapao AM, Davis SL, Barr VO, Klinker KP, Goff DA, Barber KE, et al. Large retrospective evaluation of the effectiveness and safety of ceftaroline fosamil therapy. Antimicrob Agents Chemother. (2014) 58:25416. doi: 10.1128/AAC.02371-13

41. Fabre V, Ferrada M, Buckel WR, Avdic E, Cosgrove SE. Ceftaroline in combination with trimethoprim-sulfamethoxazole for salvage therapy of methicillin-resistant Staphylococcus aureus bacteremia and endocarditis. Open Forum Infect Dis. (2014) 1:ofu046. doi: 10.1093/ofid/ofu046

42. Sakoulas G, Moise PA, Casapao AM, Nonejuie P, Olson J, Okumura $\mathrm{CY}$, et al. Antimicrobial salvage therapy for persistent Staphylococcal bacteremia using daptomycin plus ceftaroline. Clin Ther. (2014) 36:131733. doi: $10.1016 /$ j.clinthera.2014.05.061

43. Lin JC, Aung G, Thomas A, Jahng M, Johns S, Fierer J. The use of ceftaroline fosamil in methicillin-resistant Staphylococcus aureus endocarditis and deepseated MRSA infections: a retrospective case series of 10 patients. J Infect Chemother. (2013) 19:42-9. doi: 10.1007/s10156-012-0449-9

44. Britt RS, Evoy KE, Lee GC, Reveles KR, Sorensen KM, Jones X, et al. Early use of ceftaroline fosamil in the United States Veterans Health Care System. Drugs. (2017) 77:1345-51. doi: 10.1007/s40265-017-0785-2

45. Mootz ML, Britt RS, Mootz AA, Lee GC, Reveles KR, Evoy KE, et al. Comparative-effectiveness of ceftaroline and daptomycin as firstline MRSA therapy for patients with sepsis admitted to hospitals in the United States Veterans Health Care System. Hosp Pract. 47:18691. doi: 10.1080/21548331.2019.1676540

46. Tattevin P, Boutoille D, Vitrat V, Van Grunderbeeck N, Revest M, Dupont $M$, et al. Salvage treatment of methicillin-resistant staphylococcal endocarditis with ceftaroline: a multicentre observational study. J Antimicrob Chemother. (2014) 69:2010-13 doi: 10.1093/jac/dku085

47. Destache CJ, Guervil DJ, Kaye KS. Ceftaroline fosamil for the treatment of Gram-positive endocarditis: CAPTURE study experience. Int J Antimicrob Agents. (2019) 53:644-9. doi: 10.1016/j.ijantimicag.2019.01.014

48. Stucki A, Acosta F, Cottagnoud M, Cottagnoud P. Efficacy of Ceftaroline Fosamil against Escherichia coli and Klebsiella pneumoniae strains in a rabbit meningitis model. Antimicrob Agents Chemother. (2013) 57:580810. doi: 10.1128/AAC.00285-13

49. Mermer S, Turhan T, Bolat E, Aydemir S, Yamazhan T, Pullukcu H, et al. Ceftaroline versus vancomycin in the treatment of methicillin-resistant Staphylococcus aureus (MRSA) in an experimental MRSA meningitis model. $J$ Glob Antimicrob Resist. (2020) 22:147-51. doi: 10.1016/j.jgar.2020.02.001

50. Giacobbe DR, Bassetti M, De Rosa FG, Del Bono V, Grossi PA, Menichetti F, et al. Ceftolozane/tazobactam: place in therapy. Expert Rev Anti Infect Ther. (2018) 16:307-20. doi: 10.1080/14787210.2018.1447381

51. Wagenlehner FM, Umeh O, Steenbergen J, Yuan G, Darouiche RO. Ceftolozane-tazobactam compared with levofloxacin in the treatment of complicated urinary-tract infections, including pyelonephritis: a randomised, double-blind, phase III trial (ASPECT-cUTI). Lancet. (2015) 385:194956. doi: 10.1016/S0140-6736(14)62220-0
52. Solomkin J, Hershberger E, Miller B, Popejoy M, Friedland I, Steenbergen $\mathrm{J}$, et al. Ceftolozane/tazobactam plus metronidazole for complicated intraabdominal infections in an era of multidrug resistance:results from a randomized, double-blind, phase III trial (ASPECT-cIAI). Clin Infect Dis. (2015) 60:1462-71. doi: 10.1093/cid/civ097

53. Kollef MH, Nováček M, Kivistik Ü, Réa-Neto Á, Shime N, MartinLoeches I, et al. Ceftolozane tazobactam versus meropenem for treatment of nosocomial pneumonia (ASPECT-NP): a randomised, controlled, doubleblind, phase III, non-inferiority trial. Lancet Infect Dis. (2019) 19:1299311. doi: 10.1016/S1473-3099(19)30403-7

54. Yahav D, Giske CG, Grāmatniece A, Abodakpi H, Tam VH, Leibovici L. New $\beta$-Lactam- $\beta$-Lactamase inhibitor combinations. Clin Microbiol Rev. (2020) 34:e00115-20. doi: 10.1128/CMR.00115-20

55. Tato M, García-Castillo M, Bofarull AM, Cantón R, CENIT Study Group. In vitro activity of ceftolozane/tazobactam against clinical isolates of Pseudomonas aeruginosa and Enterobacteriaceae recovered in Spanish medical centres: results of the CENIT study. Int J Antimicrob Agents. (2015) 46:502-10. doi: 10.1016/j.ijantimicag.2015.07.004

56. Castanheira M, Doyle TB, Mendes RE, Sader HS. Comparative activities of ceftazidime-avibactam and ceftolozane-tazobactam against enterobacteriaceae isolates producing extended-spectrum $\beta$ Lactamases from U.S. Hospitals. Antimicrob Agents Chemother. (2019) 63:1-11. doi: 10.1128/AAC.00160-19

57. Karaiskos I, Giamarellou H. Carbapenem-sparing strategies for ESBL producers: when and how. Antibiotics. (2020) 9:61. doi: 10.3390/antibiotics9020061

58. Lodise Jr TP, Patel N, Kwa A, Graves J, Furuno JP, Graffunder E, et al. Predictors of 30-day mortality among patients with Pseudomonas aeruginosa bloodstream infections: impact of delayed appropriate antibiotic selection. Antimicrob Agents Chemother. (2007) 51:3510-5 doi: 10.1128/AAC.00 338-07

59. Shortridge D, Castanheira M, Pfaller MA, Flamm RK. Ceftolozanetazobactam activity against Pseudomonas aeruginosa clinical isolates from U.S. hospitals: report from the PACTS Antimicrobial Surveillance Program, 2012 to 2015. Antimicrob Agents Chemother. (2017) 61:e00465-17. doi: 10.1128/AAC.00465-17

60. Alatoom A, Elsayed H, Lawlor K, AbdelWareth L, El-Lababidi R, Cardona L, et al. Comparison of antimicrobial activity between ceftolozane-tazobactam and ceftazidime-avibactam against multidrug-resistant isolates of Escherichia coli, Klebsiella pneumoniae, and Pseudomonas aeruginosa. Int J Infect Dis. (2017) 62:39-43. doi: 10.1016/j.ijid.2017.06.007

61. Gonzalez MD, McMullen AR, Wallace MA, Crotty MP, Ritchie DJ, Burnham CD. Susceptibility of ceftolozane-tazobactam and ceftazidime-avibactam against a collection of b-lactam-resistant Gram-negative bacteria. Ann Lab Med. (2017) 37:174-6. doi: 10.3343/alm.2017.37.2.174

62. Maraolo AE, Mazzitelli M, Trecarichi EM, Buonomo AR, Torti C, Gentile I. Ceftolozane/tazobactam for difficult-to-treat Pseudomonas aeruginosa infections: a systematic review of its efficacy and safety for off-label indications. Int J Antimicrob Agents. (2020) 55:105891. doi: 10.1016/j.ijantimicag.2020.105891

63. Bassetti M, Castaldo N, Cattelan A, Mussini C, Righi E, Tascini C, et al. Ceftolozane/tazobactam for the treatment of serious Pseudomonas aeruginosa infections: a multicentre nationwide clinical experience. Int J Antimicrob Agents. (2019) 53:408-15. doi: 10.1016/j.ijantimicag.2018.11.001

64. Vena A, Giacobbe DR, Mussini C, Cattelan A, Bassetti M; Ceftabuse study group. Clinical efficacy of ceftolozane-tazobactam versus other active agents for the treatment of bacteremia nosocomial pneumonia due to drug resistant P. aeruginosa. Clin Infect Dis. (2020) 71:1799-801. doi: 10.1093/cid/ciaa003

65. Pogue JM, Kaye KS, Veve MP, Patel TS, Gerlach AT, Davis SL, et al. Ceftolozane/Tazobactam vs polymyxin or aminoglycoside-based regimens for the treatment of drug-resistant Pseudomonas aeruginosa. Clin Infect Dis. (2020) 71:304-10. doi: 10.1093/cid/ciz816

66. Tuon FF, Rocha JL, Formigoni-Pinto MR. Pharmacological aspects and spectrum of action of ceftazidime-avibactam: a systematic review. Infection. (2018) 46:165-81. doi: 10.1007/s15010-017-1096-y

67. Mazuski JE, Gasink LB, Armstrong J, Broadhurst H, Stone GG, Rank D, et al. Efficacy and safety of ceftazidime-avibactam plus metronidazole versus meropenem in the treatment of complicated intra-abdominal infection: 
results from a randomized, controlled, double-blind, phase III program. Clin Infect Dis. (2016) 62:1380-9. doi: 10.1093/cid/ciw133

68. Carmeli Y, Armstrong J, Laud PJ, Newell P, Stone G, Wardman A, et al. Ceftazidimeavibactam or best available therapy in patients with ceftazidime-resistant Enterobacteriaceae and Pseudomonas aeruginosa complicated urinary tract infections or complicated intra-abdominal infections (REPRISE): a randomised, pathogen-directed, phase III study. Lancet Infect Dis. (2016) 16:661-73. doi: 10.1016/S1473-3099(16)30 004-4

69. Torres A, Rank D, Melnick D, Rekeda L, Chen X, Riccobene T, et al. Randomized trial of ceftazidime-avibactam vs meropenem for treatment of hospital-acquired and ventilator-associated bacterial pneumonia (REPROVE): analyses per US FDA-Specified end points. Open Forum Infect Dis. (2019) 6:ofz149. doi: 10.1093/ofid/ofz149

70. Sousa A, Perez-Rodriguez MT, Soto A, Rodriguez L, Perez-Landeiro A, Martinez-Lamas L, et al. Effectiveness of ceftazidime/avibactam as salvage therapy for treatment of infections due to OXA-48 carbapenemaseproducing Enterobacteriaceae. J Antimicrob Chemother. (2018) 73:317075. doi: $10.1093 / \mathrm{jac} / \mathrm{dky} 295$

71. Fiore M, Alfieri A, Di Franco S, Pace MC, Simeon V, Ingoglia G, et al. Ceftazidime-Avibactam combination therapy compared to ceftazidimeavibactam monotherapy for the treatment of severe infections due to carbapenem-resistant pathogens: a systematic review and network meta-analysis. Antibiotics. (2020) 9:388. doi: 10.3390/antibiotics90 70388

72. Onorato L, Di Caprio G, Signoriello S, Coppola N. Efficacy of ceftazidime/avibactam in monotherapy or combination therapy against carbapenem-resistant Gram-negative bacteria: a meta-analysis. Int $J$ Antimicrob Agents. (2019) 54:735-40. doi: 10.1016/j.ijantimicag.2019. 08.025

73. Tumbarello M, Trecarichi EM, Corona A, De Rosa FG, Bassetti M, Mussini C, et al. Efficacy of ceftazidime-avibactam salvage therapy in patients with infections caused by Klebsiella pneumoniae Carbapenemase-producing K. pneumoniae. Clin Infect Dis. (2019) 68:355-64. doi: 10.1093/cid/c iy 492

74. Shields RK, Nguyen MH, Chen L, Press EG, Potoski BA, Marini RV, et al. Ceftazidime-avibactam is superior to other treatment regimens against carbapenem-resistant Klebsiella pneumoniae bacteremia. Antimicrob. Agents Chemother. (2017) 61:e00883-17. doi: 10.1128/AAC.00883-17

75. Corcione S, Lupia T, Maraolo AE, Mornese Pinna S, Gentile I, De Rosa FG. Carbapenem-sparing strategy: carbapenemase, treatment, and stewardship. Curr Opin Infect Dis. (2019) 32:663-73. doi: 10.1097/QCO.00000000000 00598

76. Echols R, Ariyasu M, Nagata TD. Pathogen-focused clinical development to address unmet medical need: cefiderocol targeting carbapenem resistance. Clin Infect Dis. (2019) 69(Suppl. 7):S559-64. doi: 10.1093/cid/ciz829

77. Gudiol C, Cuervo G, Carratalà J. Optimizing therapy of bloodstream infection due to extended-spectrum $\beta$-lactamase-producing Enterobacteriaceae. Curr Opin Crit Care. (2019) 25:438-48. doi: 10.1097/MCC.00000000000 00646

78. Voulgaris GL, Voulgari ML, Falagas ME. Developments on antibiotics for multidrug resistant bacterial Gram-negative infections. Expert Rev Anti Infect Ther. (2019) 17:387-401. doi: 10.1080/14787210.2019.1610392

79. Bassetti M, Ariyasu M, Binkowitz B, Nagata TD, Echols RM, Matsunaga Y, et al. Designing a pathogen-focused study to address the high unmet medical need represented by carbapenem-resistant gram-negative pathogens - the international, multicenter, randomized, open-label, Phase III CREDIBLE-CR study. Infect Drug Resist. (2019) 12:3607-23. doi: 10.2147/IDR.S225553
80. Bassetti M, Echols R, Matsunaga Y, Ariyasu M, Doi Y, Ferrer R, et al. Efficacy and safety of cefiderocol or best available therapy for the treatment of serious infections caused by carbapenem-resistant Gramnegative bacteria (CREDIBLE-CR): a randomised, open-label, multicentre, pathogen-focused, descriptive, phase III trial. Lancet Infect Dis. (2020) 21:22640. doi: 10.1016/S1473-3099(20)30796-9

81. Wunderink RG, Matsunaga Y, Ariyasu M, Clevenbergh P, Echols R, Kaye KS, et al. Cefiderocol versus high-dose, extended-infusion meropenem for the treatment of Gram-negative nosocomial pneumonia (APEKS-NP): a randomised, double-blind, phase III, non-inferiority trial. Lancet Infect Dis. (2020) 21:213-25. doi: 10.1016/S1473-3099(20)30731-3

82. Hsueh SC, Lee YJ, Huang YT, Liao CH, Tsuji M, Hsueh PR. In vitro activities of cefiderocol, ceftolozane/tazobactam, ceftazidime/avibactam and other comparative drugs against imipenem-resistant Pseudomonas aeruginosa and Acinetobacter baumannii, and Stenotrophomonas maltophilia, all associated with bloodstream infections in Taiwan. J Antimicrob Chemother. (2019) 74:380-6. doi: 10.1093/jac/dky425

83. Corcione S, Segala FV, Castiglione A, Lupia T, Angilletta R, Cavallo $\mathrm{R}$, et al. Enteropathogenetic nosocomial infections: predisposing clinical characteristics and risk of recurrent infections. J Chemother. (2019) 31:394400. doi: 10.1080/1120009X.2019.1669275

84. Plata-Menchaca EP, Ferrer R, Rodríguez JCR, Morais R, Póvoa P. Antibiotic treatment in patients with sepsis: a narrative review. Hosp Pract. (2020) 20:1-11. doi: 10.1080/21548331.2020.1791541

85. Vazquez-Grande G, Kumar A. Optimizing antimicrobial therapy of sepsis and septic shock: focus on antibiotic combination therapy. Semin Respir Crit Care Med. (2015) 36:154-66. doi: 10.1055/s-0034-1398742

86. Rhodes A, Evans LE, Alhazzani W, Levy MM, Antonelli M, Ferrer R, et al. Surviving sepsis campaign: international guidelines for management of sepsis and septic shock: 2016. Intensive Care Med. (2017) 43:30477. doi: 10.1007/s00134-017-4683-6

87. Brunkhorst FM, Oppert M, Marx G, Bloos F, Ludewig K, Putensen C, et al. Effect of empirical treatment with moxifloxacin and meropenem vs meropenem on sepsis-related organ dysfunction in patients with severe sepsis: a randomized trial. JAMA. (2012) 307:2390-99. doi: 10.1001/jama.2012.5833

88. Paul M, Lador A, Grozinsky-Glasberg S, Leibovici L. Beta lactam antibiotic monotherapy versus beta lactam-aminoglycoside antibiotic combination therapy for sepsis. Cochrane Database Syst Rev. (2014) 7:Cd003344. doi: 10.1002/14651858.CD003344.pub3

89. Ong DSY, Frencken JF, Klein Klouwenberg PMC, Juffermans N, van der Poll T, Bonten MJM, et al. Short-course adjunctive gentamicin as empirical therapy in patients with severe sepsis and septic shock: a prospective observational cohort study. Clin Infect Dis. (2017) 64:1731-6. doi: 10.1093/cid/cix186

90. Sheu CC, Chang YT, Lin SY, Chen YH, Hsueh PR. Infections caused by Carbapenem-Resistant Enterobacteriaceae: an update on therapeutic options. Front Microbiol. (2019) 10:80. doi: 10.3389/fmicb.2019.00080

Conflict of Interest: The authors declare that the research was conducted in the absence of any commercial or financial relationships that could be construed as a potential conflict of interest.

Copyright (๑) 2021 Corcione, Lupia and De Rosa. This is an open-access article distributed under the terms of the Creative Commons Attribution License (CC BY). The use, distribution or reproduction in other forums is permitted, provided the original author(s) and the copyright owner(s) are credited and that the original publication in this journal is cited, in accordance with accepted academic practice. No use, distribution or reproduction is permitted which does not comply with these terms. 